\title{
Practice Research on Application of Modern Information Technology in Network Construction of Gymnasium Management
}

\author{
Zhengxian Lv
}

\section{Huaiyin Normal University, Huaian 223001, China}

\begin{abstract}
In the information era, all kinds of information technologies are applied into various industries, playing a very important role. To adapt to needs of era development, information technology is introduced into gymnasium management. The establishment of management network becomes the content of work with great emphasis. In this paper, the author makes a research on practical application of modern information technology into the establishment of gymnasium management network.
\end{abstract}

Key words: modern information technology, gymnasium management, network construction, practical application

The application of information technology into gymnasium management is to take full advantage of computer network and perform dynamic management toward all works in gymnasium, so as to improve gymnasium management quality from a scientific perspective and maintain high-efficiency operating state as well. Gymnasium management network is established according to actual needs of gymnasium. Then, the management network system is established. In this process, the train of thought must be specified. Moreover, software and hardware equipment of computer should be properly selected at the same time. We should consider both the technical support ability of computer for gymnasium management network and the operation level of management personnel.

\section{Application of Information Technology in the Establishment of Gymnasium Management} Network

The popularization of information technology speeds up the work efficiency of various industries. Viewed from the management mode of gymnasium, it is apparent that traditional labor management fails to meet comprehensive service function of gymnasium. The application of computer information technology can realize more detailed management contents and deepen the management level. Moreover, network technology can be optimized at the same time, making it faster and more convenient for gymnasium management.

The application of information technology into gymnasium management is to utilize computer technology to integrate into digital management, implement the construction of gymnasium 
management network and improve the management efficiency by speeding up the information transfer rate. With gradually increased fitness consciousness of people and rapid development of social economy, gymnasium is no longer a place to simply meet sports events. Instead, it should give play to the function of nationwide fitness and entertainment. Therefore, many large-scale gymnasiums are developing toward the construction orientation of a comprehensive gymnasium at present. Abundant information resources make gymnasium management to be inclined to complication. Hence, considerable information resources are to be stored and rapidly arranged. The use of computer to assist gymnasium management network can remarkably improve the internal resource function of gymnasium. The development of internet technology results in rapid upgrading of computer technology, providing convenient conditions for the construction of gymnasium management network. It not only guarantees effective management of gymnasium information through the information network but also improves information safety, accuracy and effectiveness.

The application of information technology makes the gymnasium management carrier to be established on the basis of computer network. Moreover, the management pattern also changes accordingly. To give play to its advantages and meet various management needs, gymnasium should construct a local area network based on computer technology and realize network-based management. The construction of a new management system of gymnasium can make gymnasium management personnel to manage real-time information of the gymnasium. It not only gives play to the epochal character of gymnasium management but also improves the management efficiency.

\section{Application of Modern Technology in Structural Design on Gymnasium Management Network}

With the construction of a comprehensive gymnasium as an example, it is composed of multiple auxiliary stadiums, including ball training center, swimming pool and fitness center. The ground floor is for ball training. Other stadiums are arranged on the floor. For the convenience of management, both the curator's office and the accountant's office are arranged in the middle of the surface stadium and the underground stadium. The ticket window and the entrance include the surface proscenium and the underground proscenium.

(I) Application model under C/S framework

The application of C/S framework into practical network connection can receive mass data transmission. The work efficiency is high, and the safety is high as well. As the gymnasium network management system is of relatively strong information interaction, considerable information data can be transmitted within a relatively small range. Therefore, the system security 
is required to be very high. The application of C/S pattern in design of gymnasium management network system can look up the site through web browser. Moreover, clients can book site. Although C/S pattern is widely applied and applicable to flexible application sites, it lacks information interaction. B/S structure is of good expansibility, especially at client-side. It can realize non-oriented connection. During the application of model under C/S framework, favorable application effects can be obtained according to practical application and in combination with B/S structure.

(II) Database design

Microsoft SQL Server 2000 is used to design the data management system of gymnasium. It supports C/S module and B/S module. Moreover, it can effectively complete information storage and sharing at the angle of data size as well as transmission and processing of various data. SQL Server is provided with the function of data copying. It is closely integrated with the Internet, forming an open type system structure. The database platform supports graphical management, and it can realize remote system management and system configuration. While guaranteeing data integrity, it can also support storage of several processors and automatically use SQL language.

\section{Establishment of Gymnasium Management Network System based on WEB}

Generally, modern gymnasium is divided into two parts: functional area and office area. Wireless LAN is used for connection. Signals sent within dozens of meters can be received. For a large-scale comprehensive gymnasium, multiple transmitters can be used for unified terminal management.

(I) Network cabling

During the construction of gymnasium, network lines were arranged. After the connection of wireless LAN, the effective distance between router and PC is adjusted, so as to reach the overlapping state and make it convenient for users to maintain contact with network. As access point can realize roaming within the coverage area, users can be unconsciously transferred from one access point to another access point, guaranteeing smooth communication information.

(II) Distribution of network information

Under the guarantee of user roaming, wireless LAN should also consider the access head count. Generally, a wireless network access facility can support approximately 50 users. If the number of users exceeds the standard, wireless access facilities should be added accordingly.

(III) Extension of wireless network

Wireless network application includes center-free topology mode and centered topology mode. When it is necessary for network connection of computer, it only needs to install wireless fidelity to realize sharing of information resources in terms of the center-free topology mode. It can 
realize document transmission. At the same time, it can also be used as the information sending terminal and realize sharing of network resources through sub-network. During wireless access, output station can be used to control sub-station of network in terms of centered topology mode. Computer connected to wireless network can share network resources. To avoid network fault, network equipment costs should be also introduced. The combined use of wired network and wireless LAN can improve the use efficiency of gymnasium management network.

\section{Conclusion:}

To sum up, information technology is applied into the construction of modern gymnasiums to realize computer network management. As information data is of strong interaction and large exchange capacity, C/S mode is usually adopted, for the convenience of remote maintenance and improvement to safety of data transmission. Wireless LAN is the first choice for the construction of gymnasium management network at present. Internal lines can be adjusted according to specific needs of gymnasium. Moreover, it has wide coverage and relatively high freedom of use, creating convenient conditions for users.

\section{References:}

[1] Ding Wei, Research on Coordinated Management and Network Construction in Modern Logistic Combined Transport Area [D], Central South University, 2012.

[2] Dhanan jai Madhava Rao, Radharamanan Radhakrishnan, Philip A. WiIsey. Web-based

Network Analysis and Design [J]. ACM Transactions on Modeling and Computer Simulation, 2006 (10). 